\title{
Development of concurrent programs: \\ an example ${ }^{1}$
}

\author{
E. Pascal Gribomont \\ Philips Research Laboratory \\ Av. Van Becelaere 2, Box 8 \\ B - 1170 Brussels (Belgium)
}

\begin{abstract}
Concurrent programming is usually considered as a difficult task. This common opinion is investigated, while developing an example of concurrent system. The conclusion is that the difficulty of programming does not lie in concurrency, provided that adequate formal tools are used. These tools must not be more difficult than those used in sequential programming.
\end{abstract}

\section{Introduction and related work}

The formal tools to deal with concurrency are usually considered to be adequate; in fact, most of them have been derived from those used in sequential programming. Unfortunately, formal tools become most useful only when there are appropriate methodologies to deal with them. Such methodologies exist for sequential programming; for instance, Dijkstra's programming calculus [Dijkstra (1976), Gries (1981)] allows the systematic use of the concept of invariant to derive programs correct with respect to their specifications.

Good methodologies are usually compositional, that is, the meaning of a structured object can be expressed as a function of the meanings of the components. The main problem in concurrency is that the meaning of the system $(P \| Q)$ can be expressed as a function of the meanings of the processes $P$ and $Q$ only when the notion of the meaning of a process is defined in a rather complex way. As a consequence, a compositional, process-based semantics for concurrent systems is not trivial, and the same is true for the methodologies derived from those semantics [Hoare (1985)]. A solution can be to get rid of the notion of process itself, in order to keep both compositionality and simplicity. Such an approach has been recently proposed in [Chandy and Misra (1988)]. Nevertheless, the notion of process has still to be introduced at the end of the development procedure; furthermore, as the notion of process is intuitive, we would like to keep it.

Despite of the lack of a simple methodology, many non-trivial concurrent systems have been designed and proved correct. Although the authors do not often describe the methodology they have used to obtain their results, a piece of method is often evoked: the "stepwise refinement". The idea is very simple: several systems are considered in sequence, the first ones being more abstract and easier to understand than the last ones, which are more oriented towards an

\footnotetext{
${ }^{1}$ Supported in part by the ESPRIT project ATES.
} 
implementation. The notion of stepwise refinement has received several meanings, but only one is considered here. A system $A$ is a refinement of a system $B$ if every computation of $B$ is equivalent to some computation of $A$. An important particular case is when $A$ is "finer-grained" than $B$, that is, statements which were considered as atomic in $B$ are considered as composite in $A$.

A well-known example of a system developed by stepwise refinement is the "On-the-fly garbage-collector", as presented in [Dijkstra and al. (1978)]. A first, abstract version allows to understand the principle of the algorithm, whereas a second one is more complicated, but more suitable for implementation. It is observed that the invariant of the second version is also a "refinement" of the invariant of the first version. A later example is [Lamport (1983)], where several versions of a distributed system are considered. The notion of a refined invariant receives a precise definition: the refined version, if it exists, can be obtained from the initial invariant by replacing references to program variables by "state functions", that is, by expressions whose value depends not only on the value of program variables, but also on the control points of the processes. Unfortunately, the determination of the adequate state functions is not easy.

The purpose of this paper is to go a bit further, and to show how the invariant can be refined: the refined invariant appears as a solution of a set of constraints. The notion of state function is not used, but invariants are allowed to refer both to program variables and to control points.

The sequel of this paper is organized as follows. In section 2 , the programming language and the principles of the methodology are outlined, and illustrated by a toy example. A classical problem about mutual exclusion is presented in the section 3 , together with an algorithmic idea for the solution; this idea is formalized into a first, very abstract system. Some refinements are attempted in section 4 (some succeed, others fail). The properties and the implementation of the refined version are discussed in section 5 . Section 6 is a short conclusion.

\section{The programming language and the methodology}

\subsection{A simple programming language}

A system $\mathcal{S}$ consists of a set $\mathcal{P}$ of formal processes and a set $\mathcal{T}$ of transitions. A formal process, or simply a process, is a finite set of symbols called labels, or control points. A transition is an expression like

$$
T=\left(L, C \longrightarrow S, L^{\prime}\right)
$$

where $L$ is the origin, $C$ is the guard, $S$ is the statement and $L^{\prime}$ is the extremity. The type of a transition is the set of processes which are involved in it. The origin and the extremity are set of labels, containing exactly one label of each member of type(T). Let us suppose that $\operatorname{type}(T)=\{P, Q\}, L=p q$ and $L^{\prime}=p^{\prime} q^{\prime}$, where $p, p^{\prime} \in P$ and $q, q^{\prime} \in Q$. The operational semantics of $T$ is as follows. It can be executed only when processes $P$ and $Q$ are at the control 
points $p$ and $q$ respectively (this condition is represented by the formula at $p \wedge$ at $q$, or at $L$ ) and when the guard $C$ is true. The execution of the statement $S$ leads to a state where at $L^{\prime}$ is true. The guard is a predicate on the memory state of the system, and the statement specifies a transformation of this memory state.

A system $\mathcal{S}$ is considered with an invariant, that is, a formula $I$ which is respected by every transition of the system. This formula can depend not only on the memory state, but also on the control state (place predicates, like at $p$, are used to express this dependency).

A convenient piece of notation is introduced now. If $l$ is a label of a process $P$, then the formula $I_{[a t l]}$ is obtained from $I$ by replacing, for all $l^{\prime} \in P$, all occurrences of at $l^{\prime}$ by true if $l^{\prime}=l$, and by false otherwise. (The place predicates corresponding to the other processes are not replaced.) Intuitively speaking, the formula $I$ reduces to the formula $\left.I_{[a t} l\right]$ when at $l$ is assumed to be true.

The language of Labelled Transitions Systems is elementary, but powerful enough to represent most kinds of distributed systems. It is inspired from the classical concept of transition system, which is thoroughly studied in [Sifakis (1982)].

\subsection{Principle of the methodology}

The problem is to determine whether a system $\mathcal{S}$ and an invariant $I$ of this system, can be refined. For now, the only kind of refinement we consider is the splitting of a transition $T$, involving a single process $\pi=\{p, q, \ldots\}$, into two transitions $T^{\prime}$ and $T^{\prime \prime}$ involving only this process. More precisely, the transition

$$
T=(p, C \rightarrow S, q)
$$

is tentatively replaced by two new transitions

$$
\begin{aligned}
& T^{\prime}=\left(p, C \longrightarrow S^{\prime}, r\right), \\
& T^{\prime \prime}=\left(r, \text { true } \longrightarrow S^{\prime \prime}, q\right),
\end{aligned}
$$

where $r$ is a new label added into process $\pi$, and $S^{\prime}, S^{\prime \prime}$ are such that $S^{\prime} ; S^{\prime \prime}$ is sequentially equivalent to $S$. (When a guard $G$ is identically true, we write $S$ instead of $G \rightarrow S$.) It is well known that such refinements are not always valid. In fact, careless or implicit refinements are the most frequent kind of mistakes in parallel program design.

The validity of a refinement is proved as follows. If $I$ is an invariant of $\mathcal{S}$, then a formula $J$ must be obtained, such that

$$
I^{\prime}={ }_{\text {def }}[\neg a t r \Rightarrow I] \wedge[\text { at } r \Rightarrow J]
$$

is an invariant of the refined version.

The main problem is how to construct such a $J$, when it exists. The first step is to write down the constraints about $J$. These constraints are of two kinds. The first group is concerned only with the process $\pi$ and the new transitions $T^{\prime}$ and $T^{\prime \prime}$. This group consists of two sequential constraints, which are

$$
\begin{aligned}
& \left\{I_{[a t p]} \wedge C\right\} S^{\prime}\{J\}, \\
& \{J\} S^{\prime \prime}\left\{I_{[a t q]}\right\} .
\end{aligned}
$$


(It is supposed that $J=J_{[a t r]}$; this is not a restriction.)

Second, there is the group of interaction constraints, which are concerned with the other processes of the system. Every transition occurring in a process $\bar{\pi} \neq \pi$ must respect the formula $J$. For a transition $(\bar{p}, \bar{C} \rightarrow \bar{S}, \bar{q})$, the interaction condition is

$$
\left\{J_{[a t \bar{p}]} \wedge \bar{C}\right\} \bar{S}\left\{J_{[a t \bar{q}]}\right\} \text {. }
$$

The soundness of the validation method is checked easily. Let us suppose that an adequate $J$ has been found. We have to prove that all the transitions of the refined system $\mathcal{S}^{\prime}$ respect the refined invariant $I^{\prime}$. This is rather straightforward.

- Transition $T^{\prime}$. It can be executed only when at $p$ is true, that is, when at $q$ is false. In this case, $I^{\prime}$ reduces to $I$. The first sequential constraint guarantees that $J$ is true after the execution. So is at $r$, which implies that $I^{\prime}$ is true after the execution.

- Transition $T^{\prime \prime}$. It can be executed only when at $r$ is true. In this case, $I^{\prime}$ reduces to $J$. The second sequential constraint guarantees that $I$ is true after the execution. So is $\neg$ at $r$, which implies that $I^{\prime}$ is true after the execution.

- Another transition of process $\pi$. It can be executed only when at $r$ is false. In this case, $I^{\prime}$ reduces to $I$. As $I$ is respected by all transitions but $T^{\prime}$ and $T^{\prime \prime}, I$ is still true after the execution; as at $r$ is still false, $I^{\prime}$ reduces to $I$.

- A transition of another process. There are two possibilities; first, if at $r$ is false before (and after) the execution, then $I^{\prime}$ reduces to $I$ which is respected by the transition. Otherwise, it reduces to $J$ which, due to the interaction constraints, is also respected by the transition. In both cases, $I^{\prime}$ is respected.

Sequential constraints are simple, since the unknown formula $J$ occurs only once in them; on the contrary, it appears twice in the interaction constraints. For this reason, it is appropriate to solve sequential constraints first; Dijkstra's programming calculus is used to do so. These constraints determine an upper bound and a lower bound for $J$, since they can be rewritten into

$$
\begin{aligned}
& s p\left[S^{\prime} ; I_{[a t p]} \wedge C\right] \Rightarrow J, \\
& J \Rightarrow w p\left[S^{\prime \prime} ; I_{[a t q]}\right] .
\end{aligned}
$$

Formulas satisfying these implications are selected and checked against the interaction constraints. Any formula for which the verification succeeds can be adopted. Let us recall that, if the refinement is not valid, no adequate $J$ will be found. However, when the refinement is valid, the experience shows that the strongest choice, that is, the formula $s p\left[S^{\prime} ; I_{[a t} p \wedge C\right]$, often turns out to be adequate.

\subsection{Multiple refinement}

The notion of refinement has been defined to involve the introduction of a single new label into a single process. It is also possible to introduce several labels into several processes, but 
the system of constraints becomes more complicated. The experience shows that it is not interesting to introduce more than one label at a time into the same process but, if there is a symmetry between the processes, it is better not to destroy it. In this case, a refinement will involve the introduction of a new label into all the processes. As many systems of concurrent processes exhibit such a symmetry, it is interesting to consider explicitly the case of a multiple refinement.

Let $\mathcal{S}$ be a system consisting of $n$ processes $\left\{\pi_{i}: i=1, \ldots, n\right\}$ and let $\left\{T_{i}: i=1, \ldots, n\right\}$ be a set of transitions; each $T_{i}$ involves only $\pi_{i}=\left\{p_{i}, q_{i}, \ldots\right\}$. We would like to replace each $T_{i}$ by $T_{i}^{\prime}$ and $T_{i}^{\prime \prime}$ where

$$
\begin{aligned}
& T_{i}=\left(p_{i}, C_{i} \longrightarrow S_{i}, q_{i}\right), \\
& T_{i}^{\prime \prime}=\left(p_{i}, C_{i} \longrightarrow S_{i}^{\prime}, r_{i}\right), \\
& T_{i}^{\prime \prime}=\left(r_{i}, S_{i}^{\prime \prime}, q_{i}\right) .
\end{aligned}
$$

Each $r_{i}$ is a new label, introduced in $\pi_{i} ;$ it is supposed that $S_{i}^{\prime} ; S_{i}^{\prime \prime}$ is sequentially equivalent to $S_{i}$. If $I$ is an invariant of $\mathcal{S}$, then

$$
I^{\prime}=\operatorname{def}\left[\forall i\left(\neg a t r_{i}\right) \Rightarrow I\right] \wedge\left[\exists i\left(a t r_{i}\right) \Rightarrow J\right]
$$

will be an invariant of the new system $\mathcal{S}^{\prime}$ if and only if the triple

$$
\left\{I_{[a t \bar{p}]}^{\prime} \wedge \bar{C}\right\} \bar{S}\left\{I_{[a t \bar{q}]}^{\prime}\right\}
$$

is true for each transition $(\bar{p}, \bar{C} \rightarrow \bar{S}, \bar{q})$ of the system $\mathcal{S}^{\prime}$.

The strategy introduced above for simple refinements is adapted easily. It is convenient to consider first the triples whose either the precondition or the postcondition is independent from $J$. The general form of these triples is given below.

$$
\begin{array}{ll}
\left\{I_{[a t P]} \wedge C_{i}\right\} S_{i}^{\prime}\left\{J_{\left[a t P\left(p_{i} / r_{i}\right)\right]}\right\}, & i=1, \ldots, n, \\
\left\{J_{\left[a t Q\left(q_{i} / r_{i}\right)\right]}\right\} S_{i}^{\prime \prime}\left\{I_{[a t Q]}\right\}, & i=1, \ldots, n .
\end{array}
$$

The symbol $P$ denotes the sequence $p_{1} \ldots p_{n}$; the expression $P\left(p_{i} / r_{i}\right)$ denotes the sequence $p_{1} \ldots p_{i-1} r_{i} p_{i+1} \ldots p_{n}$ (the symbol $Q$ is defined in the same way). As in the simple case, these triples are solved first, by $w p$ and $s p$ calculus. Afterwards, formulas satisfying these triples are checked against the other triples (whose both the precondition and the postcondition depend on $J$ ). Any formula satisfying all the triples is an adequate choice for $J$.

Comment. Let $L$ be the sequence $\ell_{1} \ldots \ell_{n}$. The equivalence

$$
I_{[a t L]}^{\prime} \equiv I_{[a t L]}
$$

is satisfied provided that $\ell_{i} \neq r_{i}$ for all $i \in\{1, \ldots, n\} ;$ this relation is useful for the design of $I^{\prime}$.

\subsection{An elementary example}

The refinement technique is now demonstrated on a toy example: an (oversimplified) version of the "Producer-Consumer" system. 
The problem is as follows. A cyclic process $P$ repeatedly produces data which are consumed by another cyclic process $C$. Data already produced but not consumed yet are stored in a finite first-in-first-out buffer of size $N$. Obviously, no item can be consumed when the buffer is empty, and no item can be produced when it is full. An abstract solution to this problem is modelled by the following system; $\mathcal{P}$ is the family of sets of control points, and $\mathcal{T}$ is the set of transitions.

$$
\begin{aligned}
\mathcal{P}::= & \left\{\left\{P_{0}\right\},\left\{C_{0}\right\}\right\} \\
\mathcal{T}::=\left\{\left(P_{0}, p-c<N \longrightarrow \operatorname{prod}(p) ; p:=p+1, P_{0}\right)\right. & \\
& \left.\left(C_{0}, p-c>0 \longrightarrow \operatorname{cons}(c) ; c:=c+1, C_{0}\right)\right\}
\end{aligned}
$$

The variables $p$ and $c$ respectively record the number of produced data and of consumed data; $\operatorname{prod}(n)$ denotes the production of the $n$th item, whereas cons $(n)$ denotes its consumption. (These two actions do not alter the counters.) The numbers of items in the buffer is $p-c$; the buffer itself is not modelled in this representation. The invariant

$$
I^{0}=_{\text {def }} 0 \leq p-c \leq N
$$

is associated with the system. The initial conditions are represented by the formula

$$
\text { at } P_{0} \wedge \text { at } C_{0} \wedge p=c=0
$$

As a first refinement, one would like to split the production of an item and the update of the counter into two transitions. More formally, a new label $P_{1}$ is introduced in the process $P$ and the transition

$$
\left(P_{0}, p-c<N \longrightarrow \operatorname{prod}(p) ; p:=p+1, P_{0}\right)
$$

is tentatively replaced by the transitions

$$
\begin{aligned}
& \left(P_{0}, p-c<N \longrightarrow \operatorname{prod}(p), P_{1}\right) \\
& \left(P_{1}, p:=p+1, P_{0}\right)
\end{aligned}
$$

We look for an invariant $I^{1}$ of the form

$$
I^{1}=_{\text {def }}\left[\left(\neg a t P_{1} \Rightarrow I^{0}\right) \wedge\left(\text { at } P_{1} \Rightarrow K\right)\right]
$$

The constraints about $K$ are

$$
\begin{aligned}
& \left\{I_{\left[a t P_{0}\right]}^{0} \wedge p-c<N\right\} \operatorname{prod}(p)\{K\} \\
& \{K\} p:=p+1\left\{I_{\left[a t P_{0}\right]}^{0}\right\} \\
& \left\{K_{\left[a t C_{0}\right]} \wedge p-c>0\right\} \operatorname{cons}(c) ; c:=c+1\left\{K_{\left[a t C_{0}\right]}\right\}
\end{aligned}
$$

The sequential constraints can be explicited into

$$
\begin{aligned}
& (0 \leq p-c<N) \Rightarrow K, \\
& K \Rightarrow(0 \leq p+1-c \leq N)
\end{aligned}
$$

and a simple, adequate choice is $K={ }_{\text {def }}(0 \leq p-c<N)$, which leads to 


$$
I_{1}={ }_{\text {def }}\left(0 \leq p-c \wedge\left[a t P_{0} \Rightarrow p-c \leq N\right] \wedge\left[a t P_{1} \Rightarrow p-c<N\right]\right) .
$$

This choice also respects the interaction constraint.

A similar refinement is attempted for process $C$; the transition

$$
\left(C_{0}, p-c>0 \rightarrow \operatorname{cons}(c) ; c:=c+1, C_{0}\right)
$$

is tentatively replaced by the transitions

$$
\begin{aligned}
& \left(C_{0}, p-c>0 \longrightarrow \operatorname{cons}(c), C_{0}\right), \\
& \left(C_{1}, c:=c+1, C_{0}\right) .
\end{aligned}
$$

The invariant is refined further; only the result $I^{2}$ is given here.

$I^{2}={ }_{\text {def }}\left[\right.$ at $\left.P_{0} \Rightarrow p-c \leq N\right] \wedge\left[\right.$ at $\left.P_{1} \Rightarrow p-c<N\right] \wedge\left[\right.$ at $\left.C_{0} \Rightarrow p-c \geq 0\right] \wedge\left[\right.$ at $\left.C_{1} \Rightarrow p-c>0\right]$.

\section{Example - an abstract version}

As a more substantial example, the classical problem of mutual exclusion in a computer network will be considered, in a way first introduced in [Ricart and Agrawala (1981)].

\subsection{Informal description of the problem and its solution}

A network consists of $N$ nodes which communicate by messages and do not share memory. The algorithm has to create mutual exclusion between these nodes for access to a given resource. Freeness of deadlock and individual starvation must be provided.

The basic algorithmic idea is as follows. A node attempting to invoke mutual exclusion sends a request to all other nodes. On receipt of the request, the other nodes send a reply, immediate or deferred, according to a priority function. When the $N-1$ replies have been received, the access to the critical section is granted. When not immediate, a reply is deferred until the receiving node has completed its own access to the critical section.

When a station $p$ has to access its critical section, it will first indicates it and update the priority function. Afterwards, requesting messages are issued to the other stations; the access is delayed until all the answering messages have been received. The station $p$ performs some internal computation within its critical section for a while; when this computation is completed, the station $p$ sends deferred replies, if any (these replies are sent to the stations which have requested access to their own critical section). The station $p$ then comes back into its non-critical section.

The priority function is updated by a station at the very time this station begins its entry protocol. The station allows already entered stations to take precedence over it. 


\subsection{A first abstract version and its invariant}

As a very first solution, the basic idea is coded into a labelled transition system. In this most abstract version, called $\mathcal{S}_{0}$, all communication and synchronization problems are avoided. The set of processes is $\left\{\left\{p_{0}, p_{w}, p_{c}\right\}: p=1, \ldots, n\right\}$ and the transitions are

$$
\begin{aligned}
& \left(p_{0}, \neg r c s_{p} \longrightarrow[], p_{0}\right), \\
& \left(p_{0}, r c s_{p} \longrightarrow R C S_{p}:=1 ; \forall q \neq p\left[\left(P R_{p}^{q}, P R_{q}^{p}, O R_{p}^{q}\right):=(0,1,1)\right]\right. \text {; } \\
& \left.\forall q \neq p\left[\text { if } R C S_{q} \wedge P R_{q}^{p} \text { then } R D_{q}^{p}:=1 \text { else } O R_{p}^{q}:=0\right], p_{w}\right), \\
& \left(p_{w}, \forall q \neq p\left[\neg O R_{p}^{q}\right] \longrightarrow s k i p, p_{c}\right) \\
& \left(p_{c}, r c s_{p} \rightarrow[], p_{c}\right) \\
& \left(p_{c}, \neg r c s_{p} \longrightarrow R C S_{p}:=0 ; \forall q \neq p\left[\text { if } R D_{p}^{q} \text { then }\left(R D_{p}^{q}, O R_{q}^{p}\right):=(0,0)\right], p_{0}\right) \text {. }
\end{aligned}
$$

For all distinct nodes $p$ and $q$, the following variables have been used:

$R C S_{p}: p$ requests the access to the resource (Request Critical Section);

$O R_{p}^{q}: \quad p$ waits a reply from $q$ (Outstanding Reply);

$R D_{p}^{q}: \quad p$ defers a reply to $q$ (Reply Deferred);

$P R_{p}^{q}: \quad p$, on receipt of a request from $q$, takes precedence over $q$ (PRiority).

These variables will eventually be implemented as private variables but, for now, they are read and written by the stations corresponding to their indices (furthermore, $R C S_{p}$ is read by all stations). All those variables are Boolean; they are initially false. We sometimes write 1 and 0 for true and false respectively. The internal condition $r c s_{p}$ is true when station $p$ needs the access to its critical section. This condition can be set or reset only by internal computation (a step of internal computation is denoted by []; such a step alters only the internal memory of the station which performs it).

The process attached to station $p$ has three control points; at $p_{0}$ means that the station is in its non-critical section; at $p_{w}$ holds when the station is Waiting for replies and at $p_{c}$ indicates that the station is in its Critical section.

The intended meaning of the transitions is as follows.

- $\left(p_{0} \rightarrow p_{0}\right)$ models internal computation in the non-critical section.

- $\left(p_{0} \rightarrow p_{w}\right)$ models the first part of the entry protocol. Station $p$ indicates that the access to the critical section is needed, the priority function is updated and requests are made to all the other stations. The decision to grant the authorization or to delay it is taken at once, according to the priority function.

- $\left(p_{w} \rightarrow p_{c}\right)$ models waiting for access, which is the second part of the entry protocol.

- $\left(p_{c} \rightarrow p_{c}\right)$ models internal computation in the critical section.

- $\left(p_{c} \rightarrow p_{0}\right)$ models the exit protocol. The station $p$ indicates that the access to the critical section is no longer needed, and gives replies to the stations which have requested access. 
This oversimplified version of the system is not satisfactory, for the following obvious reasons.

- The communications from a station $p$ to a station $q$ are modelled by a direct transformation of the memory, performed by process $p$.

- As the priority function is used immediately after the update, that is, before any interference from another station can occur, the test $\left(R C S_{q} \wedge P R_{q}^{p}\right)$ will always reduce to $R C S_{q}$ in the transition from the initial state $p_{0}$ to the waiting state $p_{w}$.

- Large sections of program have artificially been modelled as single transitions.

These assumptions will be replaced by more realistic ones in the sequel of this paper but, first of all, the behaviour of this first version of the system has to be stated formally, in the form of an invariant. The construction of an adequate invariant is not very difficult ... since all difficulties have been artificially suppressed.

In order to obtain an invariant for the abstract system, the intended meaning of the variables is formally described by safety properties. As all variables are concerned with only one or two stations, we will look for assertions involving at most two stations, say stations $p$ and $q$.

The case of $R C S_{p}$ is very simple, since it is written by station $p$ only. At every time, exactly one of the variables $P R_{p}^{q}$ and $P R_{q}^{p}$ is true. The role of variables $O R_{p}^{q}, O R_{q}^{p}, R D_{p}^{q}$ and $R D_{q}^{p}$ is the resolution of conflicts between stations $p$ and $q$. As long as at least one of these stations is in its non-eritical state, these variables should be false. On the contrary, when both stations $p$ and $q$ are not in their non-critical state, a conflict between them has been solved: one of the station has given a reply, whereas the other has delayed its own. The latter will get (or has already got) the access before the former. This is summarized by the following set of assertions.

$$
\begin{aligned}
& \text { at } p_{0} \equiv \neg R C S_{p}, \\
& P R_{p}^{q}+P R_{q}^{p}=1 \\
& \text { at } p_{0} \Rightarrow\left(\neg O R_{p}^{q} \wedge \neg O R_{q}^{p} \wedge \neg R D_{p}^{q} \wedge \neg R D_{q}^{p}\right), \\
& \text { at } p_{w} q_{w} \Rightarrow\left(P R_{q}^{p}=O R_{p}^{q}=R D_{q}^{p} \wedge O R_{q}^{p}=R D_{p}^{q}=P R_{p}^{q}\right), \\
& \text { at } p_{w} q_{c} \Rightarrow\left(P R_{q}^{p} \wedge O R_{p}^{q} \wedge \neg O R_{q}^{p} \wedge \neg R D_{p}^{q} \wedge R D_{q}^{p}\right), \\
& \neg \text { at } p_{c} q_{c} \text {. }
\end{aligned}
$$

These assertions are true initially, since the initial conditions are formalized into the formula

$$
\text { at } p_{0} \wedge \neg R C S_{p} \wedge \neg O R_{p}^{q} \wedge \neg O R_{q}^{p} \wedge \neg R D_{p}^{q} \wedge \neg R D_{q}^{p} \wedge P R_{p}^{q}+P R_{q}^{p}=1 \text {. }
$$

We check that the conjunction of the assertions (2) is respected by every transition.

The internal transitions $\left(p_{0} \rightarrow p_{0}\right)$ and $\left(p_{c} \rightarrow p_{c}\right)$ obviously respect the set of assertions, provided that the internal statement modelled by [] does not alter the values of $R C S, P R$, $O R$ and $R D$.

If $\left(p_{0} \rightarrow p_{w}\right)$ is executed when at $q_{0}$ holds, then a sequence of assignments is executed. If we omit the assignments which do not alter the variables indexed by $p$ and $q$ only, this sequence 
reduces into $R C S_{p}:=1 ;\left(P R_{p}^{q}, P R_{q}^{p}, O R_{p}^{q}\right):=(0,1,1) ; O R_{p}^{q}:=0$, since the test $\left(R C S_{q} \wedge P R_{q}^{p}\right)$ is evaluated to false. This results into an acceptable state. If the same transition is executed in a state satisfying $a t q_{w}$ or $a t q_{c}$, then the test is evaluated to true, and the executed sequence of relevant assignments is $R C S_{p}:=1 ;\left(P R_{p}^{q}, P R_{q}^{p}, O R_{p}^{q}\right):=(0,1,1) ; R D_{q}^{p}:=1$, which leads to an acceptable state.

The transition $\left(p_{w} \rightarrow p_{c}\right)$ is tested now. When executed in a state satisfying at $q_{0}$, it leads to an acceptable state (all conflict variables are false). If it is executed in a state satisfying at $q_{w}$, then this state necessarily satisfies $O R_{p}^{q}=R D_{q}^{p}=0$ and $O R_{q}^{p}=R D_{p}^{q}=1$; this condition is respected by the transition and the resulting state is acceptable. Lastly, the same transition cannot be executed in a state satisfying at $q_{c}$, since, in such a state, the guard $O R_{p}^{q}=0$ is necessarily false.

Finally, let us test the transition $\left(p_{c} \rightarrow p_{0}\right)$. When executed in a state satisfying at $q_{0}$, then the relevant part of the executed sequence is simply $R C S_{p}:=0$, which leads to an acceptable state. If it is executed in a state satisfying at $q_{w}$, then this state satisfies $O R_{p}^{q}=R D_{q}^{p}=0$ and $O R_{q}^{p}=R D_{p}^{q}=1$. As $R D_{p}^{q}$ is true, the relevant part of the executed sequence reduces to $R C S_{p}:=0 ;\left(R D_{p}^{q}, O R_{q}^{p}\right):=(0,0)$, which leads to an acceptable state. Lastly, the same transition cannot be executed in a state satisfying at $q_{c}$, since such a state would have to satisfy the condition at $p_{c} q_{c}$.

As a conclusion, the system $\mathcal{S}_{0}$ is correct with respect to the set of assertions (2); this set is an adequate invariant $I^{0}$ for the system. In particular, mutual exclusion ( $\neg a t p_{c} q_{c}$ ), is guaranteed.

Comment. The invariant has the form

$$
\begin{aligned}
& \text { at } p_{0} \equiv \neg R C S_{p}, \\
& P R_{p}^{q}+P R_{q}^{p}=1, \\
& \forall \alpha, \beta \in\{0, w, c\}\left[\text { at } p_{\alpha} q_{\beta} \Rightarrow A(\alpha, \beta)\right] .
\end{aligned}
$$

The first assertion deals with $R C S_{p}$ which, contrary to the other variables, is updated by one process only. The second assertion is a definition of the concept of priority function; this assertion might not be altered by refinements. The last assertion is concerned with the shared variables. Every time a new label will be introduced, new formulas $A(\alpha, \beta)$ will be constructed.

\section{Refinements}

\subsection{A valid refinement}

The transition

$$
\begin{aligned}
\left(p_{0}, r c s_{p} \longrightarrow\right. & R C S_{p}:=1 ; \forall q \neq p\left[\left(P R_{p}^{q}, P R_{q}^{p}, O R_{p}^{q}\right):=(0,1,1)\right] ; \\
& \left.\forall q \neq p\left[\text { if } R C S_{q} \wedge P R_{q}^{p} \text { then } R D_{q}^{p}:=1 \text { else } O R_{p}^{q}:=0\right], p_{w}\right)
\end{aligned}
$$

contains too many actions and should be split. The first line of the transition models a preliminary phase, during which the station selects a weak priority and makes requests to all the 
other stations. The second line is the decision phase: the other stations decide whether the request will be granted at once or delayed.

A new control point $p_{d}$ (for "decision") is tentatively introduced, to split the transition $\left(p_{0} \rightarrow p_{w}\right)$ into

$$
\begin{aligned}
& \left(p_{0}, r c s_{p} \longrightarrow R C S_{p}:=1 ; \forall q \neq p\left[\left(P R_{p}^{q}, P R_{q}^{p}, O R_{p}^{q}\right):=(0,1,1)\right], p_{d}\right), \\
& \left(p_{d}, \forall q \neq p\left[\text { if } R C S_{q} \wedge P R_{q}^{p} \text { then } R D_{q}^{p}:=1 \text { else } O R_{p}^{q}:=0\right], p_{w}\right) .
\end{aligned}
$$

Comment. As the system $\mathcal{S}_{0}$ is symmetric, multiple refinements are attempted: all the processes are refined simultaneously.

The refinement gives rise to a new system $\mathcal{S}_{1}$. A new invariant $I^{1}$ has to be constructed from $I^{0}$ in order to establish the validity of this refinement. The new invariant will satisfy the equivalence

$$
I^{1} \equiv\left(\left[\forall p\left(\neg a t p_{d}\right) \Rightarrow I^{0}\right] \wedge\left[\exists p\left(a t p_{d}\right) \Rightarrow J\right]\right)
$$

This means that the old invariant $I^{0}$ must still hold every time no station $p$ is in its new control state $p_{d}$. More precisely, with the notation introduced in the paragraph 2.1 , the new invariant $I^{1}$ will satisfy the identities

$$
\forall p \forall q \neq p\left(I_{\left[a t p_{i} q_{j}\right]}^{1}=I_{\left[a t p_{i} q_{j}\right]}^{0}\right),
$$

for all $i, j$ in $\{0, w, c\}$. In addition, it is easy to check that the assertions

$$
\begin{aligned}
& \text { at } p_{0} \equiv \neg R C S_{p}, \\
& P R_{p}^{q}+P R_{q}^{p}=1 .
\end{aligned}
$$

still hold when one or both stations $p$ and $q$ are in their new control state. As a consequence, an adequate form for the new invariant $I^{1}$ is

$$
\begin{aligned}
& \text { at } p_{0} \equiv \neg R C S_{p}, \\
& P R_{p}^{q}+P R_{q}^{p}=1, \\
& \forall \alpha, \beta \in\{0, d, w, c\}\left[\text { at } p_{\alpha} q_{\beta} \Rightarrow A(\alpha, \beta)\right] .
\end{aligned}
$$

The assertions $A(\alpha, \beta)$ will be constructed as follows. First, if neither $\alpha$ nor $\beta$ is $d$, then $A(\alpha, \beta)$ is reproduced from the old invariant $I^{0}$. Second, the constraints about the remaining unknown assertions are written down; any solution of the set of constraints will lead to acceptable choices for the assertions. The common form of $A(0,0), A(0, w), A(w, 0), A(0, c)$ and $A(c, 0)$ is

whereas $A(w, w)$ is

$$
\neg O R_{p}^{q} \wedge \neg O R_{q}^{p} \wedge \neg R D_{p}^{q} \wedge \neg R D_{q}^{p}
$$

$$
\left(P R_{q}^{p}=O R_{p}^{q}=R D_{q}^{p} \wedge O R_{q}^{p}=R D_{p}^{q}=P R_{p}^{q}\right)
$$

Last, $A(w, c)$ and $A(c, w)$ are respectively

$$
\begin{aligned}
& \left(P R_{q}^{p} \wedge O R_{p}^{q} \wedge \neg O R_{q}^{p} \wedge \neg R D_{p}^{q} \wedge R D_{q}^{p}\right) \\
& \left(P R_{p}^{q} \wedge O R_{q}^{p} \wedge \neg O R_{p}^{q} \wedge \neg R D_{q}^{p} \wedge R D_{p}^{q}\right)
\end{aligned}
$$


The second step consists in writing down the set of constraints. With self-explaining notation, they can be rewritten into

$$
\begin{aligned}
& \{A(\alpha, \beta)\}\left(p_{\alpha} \rightarrow p_{\alpha^{\prime}}\right)\left\{A\left(\alpha^{\prime}, \beta\right)\right\}, \\
& \{A(\alpha, \beta)\}\left(q_{\beta} \rightarrow q_{\beta^{\prime}}\right)\left\{A\left(\alpha, \beta^{\prime}\right)\right\} .
\end{aligned}
$$

The case of the unknown assertion $A(0, d)$ is considered first. The relevant constraints for this assertion are those whose either the precondition or the postcondition is $A(0, d)$, that is

$$
\begin{aligned}
& \{A(0,0)\}\left(q_{0} \rightarrow q_{d}\right)\{A(0, d)\}, \\
& \{A(0, d)\}\left(q_{d} \rightarrow q_{w}\right)\{A(0, w)\}, \\
& \{A(c, d)\}\left(p_{c} \rightarrow p_{0}\right)\{A(0, d)\}, \\
& \{A(0, d)\}\left(p_{0} \rightarrow p_{d}\right)\{A(d, d)\} .
\end{aligned}
$$

The first two constraints are sequential constraints and can be solved easily, whereas the last two ones are interaction constraints. The sequential constraints are explicited into

$$
\begin{aligned}
& \left(\neg O R_{p}^{q} \wedge \neg R D_{p}^{q} \wedge \neg R D_{q}^{p} \wedge O R_{q}^{p}\right) \Rightarrow A(0, d), \\
& A(0, d) \Rightarrow\left(\neg R D_{p}^{q} \wedge \neg R D_{q}^{p} \wedge O R_{q}^{p}\right),
\end{aligned}
$$

and, obviously, there are only few possible choices for $A(0, d)$. The strongest formula, that is

$$
\neg O R_{p}^{q} \wedge \neg R D_{p}^{q} \wedge \neg R D_{q}^{p} \wedge O R_{q}^{p}
$$

is provisionally selected for $A(0, d)$.

Comments. A definitive choice cannot be done before all the unknown assertions have been provisionally selected, and all the interaction constraints have been checked.

The remaining three unknown assertions are selected similarly and their consistency is checked. This task can be rather fastidious, but not very difficult; in fact, most of the work could be automated. The results are

$$
\begin{array}{ll}
A(0, d): & \neg O R_{p}^{q} \wedge O R_{q}^{p} \wedge \neg R D_{p}^{q} \wedge \neg R D_{q}^{p}, \\
A(d, d): & O R_{p}^{q} \wedge O R_{q}^{p} \wedge \neg R D_{p}^{q} \wedge \neg R D_{q}^{p}, \\
A(d, w): & O R_{p}^{q} \wedge\left(O R_{q}^{p}=R D_{p}^{q}=P R_{p}^{q}\right) \wedge \neg R D_{q}^{p}, \\
A(d, c): & O R_{p}^{q} \wedge \neg O R_{q}^{p} \wedge \neg R D_{p}^{q} \wedge \neg R D_{q}^{p} \wedge P R_{q}^{p} .
\end{array}
$$

\subsection{An unsuccessful attempt}

The transition

$$
\left(p_{0}, r c s_{p} \longrightarrow R C S_{p}:=1 ; \forall q \neq p\left[\left(P R_{p}^{q}, P R_{q}^{p}, O R_{p}^{q}\right):=(0,1,1)\right], p_{d}\right)
$$

still contains too many actions; a new intermediate label $p_{i}$ is tentatively introduced, and the transition $\left(p_{0} \rightarrow p_{d}\right)$ is split into

$$
\begin{aligned}
& \left(p_{0}, r c s_{p} \longrightarrow R C S_{p}:=1, p_{i}\right) \\
& \left(p_{i}, \forall q \neq p\left[\left(P R_{p}^{q}, P R_{q}^{p}, O R_{p}^{q}\right):=(0,1,1)\right], p_{d}\right)
\end{aligned}
$$


The new invariant will have the form

$$
\begin{aligned}
& \text { at } p_{0} \equiv \neg R C S_{p}, \\
& P R_{p}^{q}+P R_{q}^{p}=1, \\
& \forall \alpha, \beta \in\{0, i, d, w, c\}\left[\text { at } p_{\alpha} q_{\beta} \Rightarrow A(\alpha, \beta)\right] .
\end{aligned}
$$

The new transitions obviously respect the first two assertions. Unfortunately, the set of constraints has no solution. As a consequence, we suspect that the refinement is not valid. In fact, an example of "bad" behaviour is easily found:

\begin{tabular}{cccccccc}
$p$ & $q$ & $O R_{p}^{q}$ & $O R_{q}^{p}$ & $R D_{p}^{q}$ & $R D_{q}^{p}$ & $P R_{p}^{q}$ & $P R_{q}^{p}$ \\
\hline$p_{i}$ & $q_{i}$ & 0 & 0 & 0 & 0 & 1 & 0 \\
$p_{i}$ & $q_{d}$ & 0 & 1 & 0 & 0 & 1 & 0 \\
$p_{i}$ & $q_{w}$ & 0 & 1 & 1 & 0 & 1 & 0 \\
$p_{d}$ & $q_{w}$ & 1 & 1 & 1 & 0 & 0 & 1 \\
$p_{w}$ & $q_{w}$ & 1 & 1 & 1 & 1 & 0 & 1
\end{tabular}

The system is deadlocked since both stations will wait forever a reply from each other. This failure does not mean that transition $\left(p_{0} \rightarrow p_{d}\right)$ cannot be split, but that it cannot be split in the way we have just attempted.

\subsection{A medium-grained version}

Further refinements are attempted following the same method. Some will succeed, other will fail. As the development is very similar to that presented in the previous paragraph, only the resulting system and its invariant are given.

The system $\mathcal{S}_{2}$ contains the following transitions, for all $p$.

$$
\begin{aligned}
& \left(p_{0}, \neg r c s_{p} \longrightarrow\left[1, p_{0}\right),\right. \\
& \left(p_{0}, r c s_{p} \longrightarrow R C S_{p}:=1 ; \forall q \neq p\left[\left(P R_{p}^{q}, P R_{q}^{p}\right):=(0,1)\right], p_{1}\right) \\
& \left(p_{1}, \forall q \neq p\left[O R_{p}^{q}:=1\right], p_{2}\right) \\
& \left(p_{2}, \forall q \neq p\left[\text { if } R C S_{q} \wedge P R_{q}^{p} \text { then } R D_{q}^{p}:=1 \text { else } O R_{p}^{q}:=0\right], p_{3}\right) \\
& \left(p_{3}, \forall q \neq p\left[\neg O R_{p}^{q}\right] \rightarrow \text { skip, } p_{4}\right) \\
& \left(p_{4}, r c s_{p} \longrightarrow[], p_{4}\right), \\
& \left(p_{4}, \neg r c s_{p} \longrightarrow R C S_{p}:=0, p_{5}\right) \\
& \left(p_{5}, \forall q \neq p\left[\text { if } R D_{p}^{q} \text { then }\left(R D_{p}^{q}, O R_{q}^{p}\right):=(0,0)\right], p_{0}\right) .
\end{aligned}
$$

For the sake of uniformity, the labels $p_{d}, p_{w}$ and $p_{c}$ have been renamed $p_{2}, p_{3}$ and $p_{4}$ respectively; $p_{1}$ and $p_{5}$ are new labels. The invariant $I^{2}$ can be concisely written as follows.

$$
\begin{aligned}
& \left(\text { at } p_{0} \vee \text { at } p_{5}\right) \equiv \neg R C S_{p}, \\
& \left(P R_{p}^{q}+P R_{q}^{p}=1\right) \wedge\left(\left(\text { at } p_{4} \wedge R C S_{q}\right) \Rightarrow P R_{p}^{q}\right), \\
& \left(\text { at } p_{0} \vee \text { at } p_{1} \vee \text { at } p_{4} \vee \text { at } p_{5}\right) \Rightarrow \neg O R_{p}^{q}, \\
& \text { at } p_{2} \Rightarrow O R_{p}^{q}, \\
& \left(\text { at } p_{3} \wedge \neg \text { at } q_{5}\right) \Rightarrow O R_{p}^{q}=\left[\left(\text { at } q_{1} \vee \text { at } q_{2} \vee \text { at } q_{3} \vee \text { at } q_{4}\right) \wedge P R_{q}^{p}\right] \\
& O R_{p}^{q}=\left(\text { at } p_{2}+R D_{q}^{p}\right)
\end{aligned}
$$




\section{Properties and implementation of the refined version}

\subsection{Deadlock and starvation}

The invariant expresses that the refined version guarantees the mutual exclusion, but this property is not sufficient. In order to be of practical use, the system should also be free from deadlock and starvation. Due to the invariant, two stations $p$ and $q$ could not be locked in their waiting state, waiting for a reply from each other. However, the invariant still allows the following state, concerning three distinct stations $p, q$ and $r$ :

$$
\text { at } p_{3} \wedge \text { at } q_{3} \wedge \text { at } r_{3} \wedge P R_{p}^{q} \wedge P R_{q}^{r} \wedge P R_{\tau}^{p} \text {. }
$$

In such a state, station $r$ is waiting for station $q$, which is waiting for station $p$, which is waiting for station $r$. None of these three stations will ever gain access, and, in fact, all the other stations will be also delayed forever.

It is possible to strengthen the invariant in order to show that no "cyclic" blocking state can be reached. Let $D$ be any subset $\left\{a_{1}, \ldots, a_{m}\right\}$ of the set $\{1, \ldots, n\}$. A D-blocking state is a state satisfying a formula $F(D)$ defined as

$$
P R_{a_{1}}^{a_{2}} \wedge \cdots \wedge P R_{a_{m-1}}^{a_{m}} \wedge P R_{a_{m}}^{a_{1}}
$$

It is easy to check that the execution of the transition $\left(p_{0} \rightarrow p_{1}\right)$, if started in a non-blocking state, always results in a non-blocking state. More precisely, we have to check the validity of the triple

$$
\{\forall D \subset\{1, \ldots, n\}[\neg F(D)]\}\left(p_{0} \rightarrow p_{1}\right)\{\forall D \subset\{1, \ldots, n\}[\neg F(D)]\} .
$$

If $p \notin\left\{a_{1}, \ldots, a_{m}\right\}$, then the transition has no effect on formula $F(D)$. If $p$ is, say, $a_{1}$, then the resulting state satisfies the formulas $P R_{j}^{a_{1}}$ for all $j$, and station $p=a_{1}$ cannot belong to any cycle. The mutual priorities between the other stations are left as before the execution, that is, without cycle.

\subsection{Implementation as a set of communicating processes}

As all variables of the system are altered by at most two stations, the transformation of the system $\mathcal{S}_{2}$ into a network of communicating processes should be easy. However, there is still a critical point. The transitions $\left(p_{0} \rightarrow p_{1}\right),\left(p_{2} \rightarrow p_{3}\right)$ and $\left(p_{5} \rightarrow p_{0}\right)$ involve message passing between stations. As these transitions have not been refined further, all the communications between a station and all the others should be simultaneous. For instance, when station $p$ sends a message to the other stations to take the lowest priority, all the other stations must receive these messages without sending or receiving any other messages. This policy requires a high degree of synchronism and is therefore rather inefficient.

A solution could be the further refinement of these communication transitions but, unfortunately, the refinement of the transition $\left(p_{0} \rightarrow p_{1}\right)$ would introduce risks of deadlock. Another 
solution, adopted in [Ricart and Agrawala (1981)], consists in a slight modification of the priority function, which allows these authors to obtain an implementation of the system as a set of communicating processes. The formal presentation and justification of this implementation is too long to be presented here, but the main point is the fact that the invariant (6) remains valid (even about the priority function). More details are given in [Gribomont (1985)].

\section{Conclusion}

The purpose of this paper was to demonstrate that the development of parallel program can be carried out with simple formal tools. Let us now summarize where are, and where are not, the critical points of the development. The most difficult point is the discovery of a good initial algorithmic idea. The original idea of Ricart and Agrawala can be explained in a simple way, but is not so simple (otherwise, it would have been discovered earlier). On the contrary, the final solution to the problem, or even the intermediate solution $\mathcal{S}_{2}$ presented in the previous section, seems difficult when considered in isolation. It becomes easy to understand when earlier versions, with their invariants, are considered first. (Another critical point, which has not been described in this paper, is the discovery of a priority function fully appropriate to a distributed implementation.)

As a conclusion, the main critical point in concurrent programming is exactly the same that in sequential programming, i.e., the discovery of new algorithmic ideas. The development of the programs based on these ideas is comparatively simple, provided that an adequate development method is used. The problem is that the use of an adequate method is not frequent in programming; this is especially dangerous when concurrency is introduced.

\section{References}

[1] K.M. CHANDY and J. MISRA, "Parallel Program Design: A Foundation", Addison-Wesley, 1988.

[2] E.W. DIJKSTRA, “A discipline of programming", Prentice Hall, New Jersey, 1976.

[3] E.W. DIJKSTRA and al., "On-the-Fly Garbage Collection: An Exercise in Cooperation", CACM, vol. 21, pp. $966-975,1978$.

[4] E.P. GRIBOMONT, "Méthode progressive de synthèse et de vérification de programmes parallèles", Thèse de doctorat, Université de Liège, 1985.

[5] D. GRIES, "The Science of Programming", Springer-Verlag, Berlin, 1981.

[6] C.A.R. HOARE, "Communicating Sequential Processes", CACM, vol. 21, pp. 666-677, 1978.

[7] C.A.R. HOARE, "Communicating Sequential Processes", Prentice-Hall, 1985.

[8] L. LAMPORT, "An Assertional Correctness Proof of a Distributed Algorithm", Science of Computer Programming, vol. 2, pp. 175-206, 1983.

[9] G. RICART and A.K. AGRAWALA, "An optimal algorithm for mutual exclusion", CACM, vol. 24, pp. 917, 1981 (corr. vol. 24, p. 578).

[10] J. SIFAKIS, "A unified approach for studying the properties of transition systems", Theoretical Computer Science, vol. 18, pp. 227-259, 1982. 